\title{
Apendicite aguda em paciente idoso: relato de caso
}

Acute appendicitis in elderly patient: case report

Apendicitis aguda en paciente mayor: reporte de caso

Heitor Rubio VERGINIO'

Amanda Oliva SPAZIANI 1

Arlindo Mariano de ARAÚJO NETO 1

Geisa Peixoto CARDOSO ${ }^{1}$

Trícia Aline Ribeiro Pattini de SOUZA²

Gustavo Rivelli LAMBOGLIA2

Rodrigo João Lopes TAVEIROS²

Marcos Rogério MARQUES ${ }^{2}$

Ana Carolina Borges GORGA 3

Márcio Vinicius Dores RENESTO3

Leonardo FAIDIGA3

Pedro Gomes BARBOSA JUNIOR ${ }^{3}$

${ }^{I}$ Curso de Medicina, Universidade Brasil Fernandópolis, São Paulo, Brasil

${ }^{2}$ Residente de Cirurgia Geral do Hospital Ensino Santa Casa de Misericórdia de Fernandópolis, São Paulo, Brasil ${ }^{3}$ Médico cirurgião geral

\section{Resumo}

O apêndice vermiforme é um órgão tubular de fundo cego que se origina da parede do ceco. A apendicite é a causa mais comum de dor abdominal aguda e requer intervenção cirúrgica. Paciente masculino, 70 anos, com queixa de dor abdominal difusa tipo cólica de início há 3 dias e há 2 dias relata parada da evacuação e flatos. Durante o exame abdominal constatou-se abdome plano, levemente distendido, com ruídos hidroaéreos presentes, hipertimpânico à palpação, doloroso a palpação de forma difusa, com sinal de Rovsing positivo. Os sinais de Blumberg, Dunphy, Lapinsky, Iliopsoas Obturador, Giordano e Murphy negativos. Ao Toque retal, próstata ligeiramente aumentada. Aos exames laboratoriais: hemograma com leucocitose. Aos exames de imagem raio-x de abdome agudo, tomografia de abdome total e transito intestinal: distensão gasosa de alças intestinais com níveis hidroaéreos e ausência de líquido livre. Submetido a laparatomia exploratória que evidenciou distensão de intestino delgado e afunilamento de cólons transverso, descendente e sigmoide com apêndice cecal supurado grau 5 e grande quantidade de aderências em alças intestinais. Realizada apendicectomia e iniciada antibioticoterapia de amplo expectro. Obteve alta hospitalar após melhora da distensão e quadro infeccioso. A apendicite é normalmente uma condição patológica de jovens. Entretanto, o aumento da expectativa de vida tornou a ocorrência de apendicite aguda em pacientes idosos mais comum. A mortalidade da apendicite aguda no paciente idoso é mais elevada. Dessa forma, pacientes idosos apresentam pior prognostico, maior tempo de evolução da doença, maior permanência no hospital e mais casos de perfuração.

Descritores: Apendicite; Abdome Agudo; Idoso.

\section{Abstract}

The vermiform appendix is a blind-bottomed tubular organ that originates from the cecum wall. Appendicitis is the most common cause of acute abdominal pain and requires surgical intervention. A 70-year-old male patient complaining of diffuse colic-type abdominal pain that began 3 days ago and 2 days ago reported stopping the bowel movement and flatus. During the abdominal examination, a slightly distended flat abdomen was observed, with watery airborne noises, hyper-panic on palpation, diffuse painful palpation, with a positive Rovsing sign. The signs of Blumberg, Dunphy, Lapinsky, Iliopsoas Shutter, Giordano and Murphy negative. At rectal touch, prostate slightly enlarged. Laboratory tests: blood count with leukocytosis. X-ray imaging of acute abdomen, total abdomen tomography and intestinal transit: gas distension of the intestinal loops with airborne levels and absence of free fluid. The patient underwent exploratory laparatomy that showed small bowel distension and transverse, descending, and sigmoid colonic funnel with grade 5 cecal appendage and a large amount of adhesions in the intestinal loops. Appendectomy was performed and broad-spectrum antibiotic therapy started. He was discharged from hospital after improvement of distension and infectious condition. Appendicitis is usually a pathological condition of young people. However, increased life expectancy has made the occurrence of acute appendicitis in elderly patients more common. Mortality from acute appendicitis in the elderly patient is higher. Thus, elderly patients have worse prognosis, longer disease progression, longer hospital stay and more perforation cases.

Descriptors: Appendicitis; Abdomen, Acute; Aged.

\section{Resumen}

El apéndice vermiforme es un órgano tubular de fondo ciego que se origina en la pared del ciego. La apendicitis es la causa más común de dolor abdominal agudo y requiere intervención quirúrgica. Un paciente masculino de 70 años que se queja de dolor abdominal difuso de tipo cólico que comenzó hace 3 días y hace 2 días informó haber interrumpido el movimiento intestinal y los flatos. Durante el examen abdominal, se observó un abdomen plano ligeramente distendido, con ruidos acuosos en el aire, hiperpánico al palpar, palpación difusa dolorosa, con un signo positivo de Rovsing. Los signos de Blumberg, Dunphy, Lapinsky, Iliopsoas Shutter, Giordano y Murphy son negativos. En contacto rectal, próstata ligeramente agrandada. Pruebas de laboratorio: hemograma con leucocitosis. Radiografía de abdomen agudo, tomografía total de abdomen y tránsito intestinal: distensión de gas de las asas intestinales con niveles en el aire y ausencia de líquido libre. El paciente se sometió a una laparatomía exploratoria que mostró distensión del intestino delgado y embudo de colon transverso, descendente y sigmoide con apéndice cecal de grado 5 y una gran cantidad de adherencias en las asas intestinales. Se realizó apendicectomía y se inició tratamiento antibiótico de amplio espectro. Fue dado de alta del hospital después de mejorar la distensión y la condición infecciosa. La apendicitis suele ser una afección patológica de los jóvenes. Sin embargo, el aumento de la esperanza de vida ha hecho más frecuente la aparición de apendicitis aguda en pacientes de edad avanzada. La mortalidad por apendicitis aguda en el paciente anciano es mayor. Por lo tanto, los pacientes de edad avanzada tienen peor pronóstico, mayor progresión de la enfermedad, mayor estadía en el hospital y más casos de perforación.

Descriptores: Apendicitis; Abdomen Agudo; Anciano.

\section{INTRODUÇÃO}

O apêndice vermiforme é um órgão tubular de fundo cego que se origina da parede pósteromedial do ceco, localizado a aproximadamente $2 \mathrm{~cm}$ abaixo da válvula ileocecal, com comprimento entre
2 a $20 \mathrm{~cm}$, paredes apresentam com espessura de aproximadamente $3 \mathrm{~mm}$ e continuas com as camadas do ceco, mucosa, submucosa, muscular e serosa, porém possuem uma submucosa com mais tecido 
linfático. Histologicamente, o apêndice segue o padrão do ceco. Notadamente, na sua submucosa, encontramos folículos linfáticos cujo número varia com a idade, aumentando desde a infância e podendo chegar a 200 na faixa etária situada entre 10 e 20 anos. O número de folículos vai diminuindo progressivamente, principalmente após os 30 anos, podendo ser totalmente ausentes após os 60 anos. $\mathrm{O}$ número de folículos tem grande impacto na maior incidência de apendicite em jovens ${ }^{1}$.

A apendicite é a causa mais comum de dor abdominal aguda, que requer intervenção cirúrgica. Classicamente, a obstrução do apêndice vermiforme por fecalito, corpo estranho, parasitas, hiperplasia linfonodal e tumores, tem sido relacionada ao desenvolvimento da apendicite aguda. Nesta teoria, a apendicite aguda é considerada uma condição progressiva, que se inicia com o aumento na secreção de muco e a elevação da pressão intraluminal, resultando em estase venosa, compressão arterial e isquemia das paredes do órgão. Com a isquemia da mucosa, o mecanismo protetor de barreira é perdido, levando à invasão bacteriana da parede do apêndice, que por sua vez favorece o infarto e a perfuração do apêndice ${ }^{2}$.

Entretanto, a observação da resolução espontânea de casos de apendicite aguda e os relatos por parte de alguns autores da boa evolução em casos tratados com antibiótico mostram que nem todos os casos de apendicite aguda enquadram-se na teoria da obstrução mecânica e progressão para apendicite complicada. Seu diagnóstico é habitualmente fácil e essencialmente clínico, porém, podem ocorrer algumas apresentações atípicas, que são relacionadas, principalmente, à localização do apêndice retrocecal e pélvica, que tornam o diagnóstico da apendicite um desafio clínico-cirúrgico. A sintomatologia clássica predomina em $60 \%$ dos casos. A eficácia em diagnosticar apendicite aguda é de cerca de $80 \%$, sendo de 78-92\% em homens e de 58- $85 \%$ em mulheres. O diagnóstico diferencial e as variações anatômicas representam os principais entraves em seu reconhecimento clínico, o que propicia atraso em relação ao diagnóstico e tratamento e aumento das chances de complicação ${ }^{3}$.

Apresenta incidência de 48,1 por dez mil habitantes por ano, e seu pico de incidência ocorre em pacientes entre os 10 e 20 anos de idade. $\mathrm{O}$ risco geral ao longo da vida é estimado entre $5 \%$ e $20 \%$, sendo de $8,6 \%$ para homens e 6,7\% para mulheres. Afeta aproximadamente 250 mil pacientes por ano nos EUA e é responsável por pelo menos $40 \mathrm{mil}$ admissões hospitalares, por ano, na Inglaterra ${ }^{2,4}$.

\section{CASO CLÍNICO}

Paciente do sexo masculino, 70 anos de idade, com queixa de dor abdominal difusa tipo cólica de início há 3 dias, sem fatores de melhora e piora, sem irradiação e sem alterações, refere episódio de febre não aferida e hiporexia associada há 3 dias. Há 2 dias relata parada da evacuação e flatos, além de oligúria. Realizando uso de tramadol endovenoso para quadro álgico com melhora. Refere obstipação crônica, nega doenças previas. É tabagista há 45 anos, ex-etilista e ex-usuário de drogas ilícitas. Ao exame físico apresentou-se desidratado, descorado, anictérico, acianótico, taquipneico (FR = 26 irpm) e febril (38,8 graus Celsius). Ao exame cardiovascular apresentava bulhas rítmicas, normofonéticas, em 2 tempos, sem sopros, $\mathrm{FC}=115$ bpm. No exame respiratório o murmúrio vesicular era presente bilateralmente sem ruídos adventícios. Durante o exame abdominal constatou-se abdome plano, levemente distendido, com ruídos hidroaéreos presentes, hipertimpânico à palpação, doloroso a palpação de forma difusa, com sinal de Rovsing positivo. Os sinais de Blumberg, Dunphy, Lapinsky, Iliopsoas Obturador, Giordano e Murphy negativos. Ao Toque retal, próstata ligeiramente aumentada. Aos exames laboratoriais: hemograma com leucocitose e exame de urina infeccioso. Aos exames de imagem raio-x de abdome agudo, tomografia de abdome total e transito intestinal: distensão gasosa de alças intestinais com níveis hidroaéreos e ausência de líquido livre.

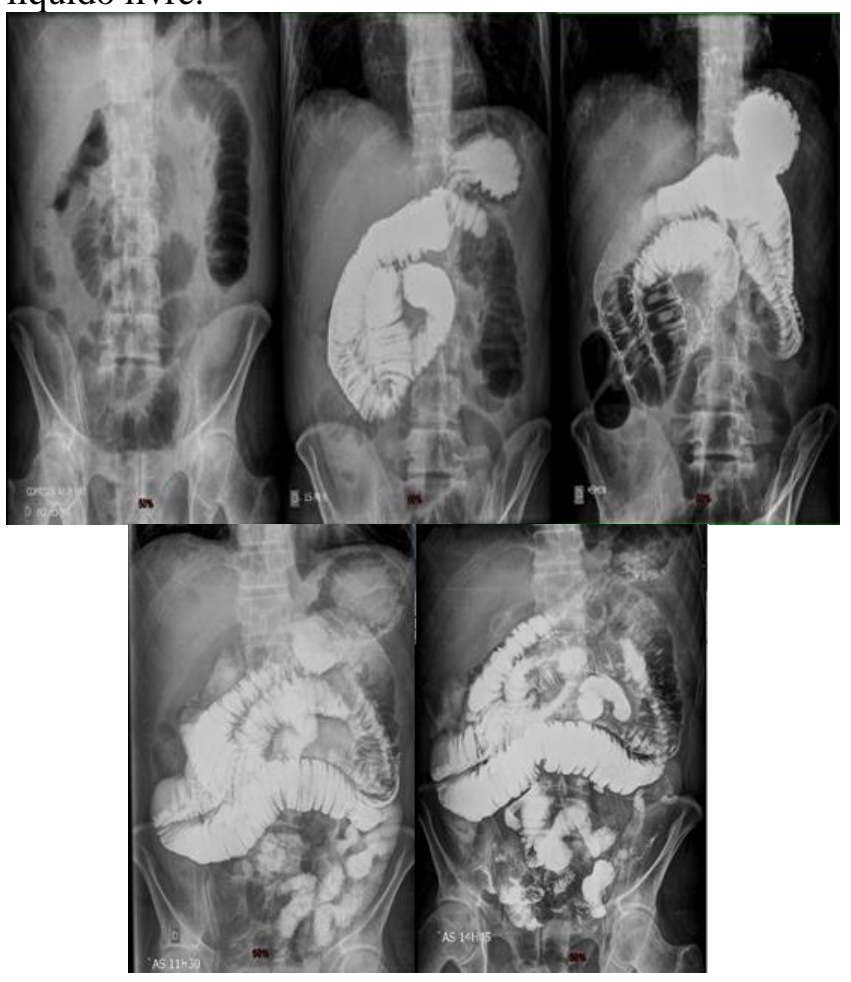

Figuras 1 a 5: Radiografias de trânsito intestinal apresentando: distensão gasosa de alças intestinais. Trânsito gastro-intestinal lento, com distensão importante de alças intestinais. Houve contrastação de alças ileais após 3 horas e 30 minutos. Não se observou passagem de contraste para o ceco até a grafia de 7 horas. FONTE: exames cedidos pelo paciente. FONTE: realizadas pelos médicos responsáveis.

Submetido a laparatomia exploratória que evidenciou distensão de intestino delgado e afunilamento de cólons transverso, descendente e sigmoide com apêndice cecal supurado grau 5 e grande quantidade de aderências em alças intestinais. Realizada apendicectomia e iniciada 
antibioticoterapia de amplo espectro. Boa evolução, porém lenta, com leve distensão abdominal e transito intestinal lento. Obteve alta hospitalar após melhora da distensão e quadro infeccioso.

DISCUSSÃO

A apendicite é normalmente uma condição patológica de jovens. Entretanto, o aumento da expectativa de vida populacional com uma pirâmide etária com o ápice alargado mostrando uma população cada vez mais idosa, tornou a ocorrência de apendicite aguda em pacientes acima de 40 ou 60 anos mais comum. $\mathrm{O}$ diagnóstico clínico apropriado nos pacientes idosos é frequentemente complicado por muitos fatores que levam a um quadro clínico atípico da doença, eles incluem diminuição da função imunológica, sintomas vagos, múltiplas comorbidades e alterações anatômicas normais relacionadas a idade. Isso é atribuído a apresentações atípicas que levam a frequentes erros de diagnóstico. Aceito o fato de que o quadro clinico das complicações da apendicite aguda no paciente idoso pode diferir no jovem, torna-se necessária uma análise minuciosa em particular desse grupo de pacientes $^{5,6}$.

Embora seja uma afecção que ocorra principalmente no paciente adulto jovem, a apendicite aguda também pode comprometer pacientes de qualquer idade. Embora essa incidência seja incomum, a apendicite aguda no idoso se mostra relevante, visto a maior morbidade e mortalidade em relação ao jovem. A mortalidade da apendicite aguda no paciente idoso é mais elevada, variando de cinco a dezesseis vezes em relação ao jovem, isso pode estar relacionado ao quadro clinico atípico, o que retarda o diagnóstico e consequentemente causa maior postergação no tratamento cirúrgico. Sabe-se que o intervalo entre o início dos sintomas e a internação para os octogenários foi quase $\mathrm{o}$ dobro $\mathrm{em}$ comparação com os pacientes mais jovens ${ }^{3}$.

$\mathrm{O}$ maior número de complicações em pacientes idosos é explicado pelo fato de serem comumente portadores de doenças sistêmicas associadas, principalmente cardíacas e respiratórias, o que predispõe a descompensarão e desencadeia maior número de complicações pré-operatórias, intraoperatórias e pós-operatórias, principalmente nos casos de apendicite complicada. O maior número de complicações locais ou sistêmicas neste grupo de pacientes reflete no maior número de permanência hospitalar pós-operatória ${ }^{4}$.

Dessa forma, pacientes idosos apresentam pior prognostico, maior tempo de evolução da doença, maior permanência no hospital e mais casos de perfuração apendicular. Entretanto, apesar de alguns autores consentirem que as complicações tendem a ser maiores no paciente idoso, consideram que a perfuração apendicular no idoso não é só devido ao diagnóstico tardio, consequente a uma sintomatologia atípica, mas também a uma disseminação mais rápida da inflamação ${ }^{4}$.

A progressiva aceleração do processo seria devido não só a particularidades anatômicas do apêndice cecal, como também da esclerose da artéria apendicular e fraqueza estrutural da parede do apêndice, atribui-se, ainda, uma diminuição dos mecanismos de defesa e reação tecidual. Seu diagnóstico precoce é essencial para reduzir a morbidade. $\mathrm{O}$ advento dos antibióticos e o manejo cirúrgico eficiente têm reduzido substancialmente a sua mortalidade, entretanto, ainda se associa à morte, particularmente em idosos? ${ }^{7}$.

\section{REFERÊNCIAS}

1. Tamburrini S, Brunetti A, Brown M, Sirlin CB, Casola G. CT appearance of the normal appendix in adults. Eur radiol. 2005;15(10):2096-103.

2. Santos F, Cavasana GF, Campos T. Perfil das apendicectomias realizadas no Sistema Público de Saúde do Brasil. Rev Col Bras Cir. 2017;44 (1):4-8.

3. Gomes CA, Nunes TA. Classificação laparoscópica da apendicite aguda: correlação entre graus da doença $\mathrm{e}$ as variáveis perioperatórias. Rev Col Bras Cir. 2006;33(5): 289-93.

4. Freitas RG, Pitombo MB, Maya MCA, Leal PRF. Apendicite aguda. Rev Hosp Univ Pedro Ernesto. 2009;8(1):38-51.

5. Storm-Dickerson TL, Horattas MC: What we have learned over the past 20 years about appendicitis in the elderly? Am J Surg. 2003;185(3)198-201.

6. Brenner AS, Santin J, Virmond Neto F, Boursheid $\mathrm{T}$, Valarini R, Rydygier R. Apendicectomia em pacientes com idade superior a 40 anos: análise dos resultados de 217 casos. Rev bras Coloproct. 2006; 26(2):128-32.

7. Almeida MWR, Trevisan JA, Oliveira FS, Mattos HC, Silva AR, Barbosa e Silva MCG. Influência da idade no tempo de internação e grau evolutivo das apendicites agudas: uma revisão em um Hospital Universitário do sul do país. Rev Col Bras Cir. 2006;33(5):294-97.

\section{CONFLITO DE INTERESSES}

Os autores declaram não haver conflitos de interesse.

\section{AUTOR PARA CORRESPONDENCIA}

\section{Amanda Oliva Spaziani}

spazianimedicina@gmail.com 